\title{
Distributed Classification of Traffic Anomalies using Microscopic Traffic Variables
}

\author{
S. Thajchayapong, E. S. Garcia-Treviño and J. A. Barria
}

\begin{abstract}
This paper proposes a novel anomaly classification algorithm that can be deployed in a distributed manner and utilizes microscopic traffic variables shared by neighbouring vehicles to detect and classify traffic anomalies under different traffic conditions. The algorithm which incorporates multi-resolution concepts is based on the likelihood estimation of a neural network output and a bisection-based decision threshold. We show that when applied to real-world traffic scenarios, the proposed algorithm can detect all the traffic anomalies of the reference test data set; this represents a significant improvement over our previously proposed algorithm [1]. We also show that the proposed algorithm can effectively detect and classify traffic anomalies even when i) the microscopic traffic variables are available from only a fraction of the vehicle population and ii) some microscopic traffic variables are lost due to degradation in V2V and/or V2I communications.
\end{abstract}

Keywords: Distributed traffic monitoring, traffic anomalies detection, incident precursors, microscopic traffic variables, freeway segments, V2V, V2I.

\section{INTRODUCTION}

The first step in detecting the possible emergence of traffic incidents is to identify traffic anomalies, which in this paper are defined as traffic patterns that do not conform to expected behaviour [1-3]. Once the detected abnormal traffic pattern has been identified, information regarding its potential impacts can be disseminated to individual drivers and to traffic management centres, such as Advanced Traveler Information System (ATIS) and Advanced Traffic Management System (ATMS), so that the appropriate proactive strategies to minimize the response times, clear the roadways, and recover traffic condition back to normality can be promptly set in place.

Recent advances in vehicle-to-vehicle (V2V) and vehicle-to-infrastructure (V2I) wireless communications have increased the potential of real-time measuring and processing of microscopic traffic variables from vehicles

S. Thajchacyapong is with National Electronic and Computer Technology Center (NECTEC), National Science and Technology Development Agency (NSTDA), Thailand. E. S. Garcia-Treviño and J. A. Barria are with the Department of Electrical and Electronic Engineering, Imperial College London, United Kingdom. contact email: j.barria@imperial.ac.uk in a distributed manner. Distributed monitoring in this paper refers to the process by which traffic anomalies are detected and classified by local vehicles themselves and/or road-side infrastructure based on local traffic information on the road segment of interest, without the need to send traffic information to a traffic management centre. We will refer to these local vehicles and/or roadside infrastructure as nodes in the remainder of this paper.

Recent studies have attempted to develop algorithms to detect traffic anomalies that lead to traffic disruptions, commonly referred as incident precursors [4-6], an incident being a non-recurring major traffic disruption whose occurrence is usually unexpected and random [7]. However, since most of these algorithms are based on macroscopic traffic variables derived only from road-side infrastructure, such as loop detectors, their effectiveness largely depends on the relative location of the anomaly in respect to the loop detectors. If a disruption takes place far from the loop detector location, the anomaly may not be detected or a long delay may occur before the anomaly is identified. On the other hand, microscopic traffic information obtained from vehicles has the advantages of coverage and sensitivity to support distributed anomaly detection schemes. The results presented in this paper as well as previous findings $([1,8])$, strongly suggest that algorithms capable of using available microscopic traffic variables for anomaly detection and classification are worth developing. In this paper we present a novel algorithm capable of detecting traffic anomalies in a distributed manner.

In order to use microscopic traffic information in a distributed manner, an algorithm has to at least possess the following properties: first, the algorithm needs to be deployable on a local node where there may be limited local storage and communication capabilities. The second property is that an anomaly detection and classification algorithm not only needs to detect different types of traffic anomalies but should also minimize false alarm rate (FAR) and hence reduce emergency response costs. Furthermore, an early warning signal has to be available as fast as possible to give enough 
lead time to operations/management personnel to initiate appropriate emergency responses before the anomaly evolves into a major traffic disruption. Here we note that previously proposed algorithms have been assessed on maximizing detection rate (DR) but not properly tested on FAR and mean time to detection (MTTD) [4]. Finally, the algorithm should be adaptable to different traffic conditions such as, e.g. a low-flow low-speed condition during rush hours and a low-flow high-speed condition during daytime periods. Most of the existing algorithms use a pre-specified time window $(5 \mathrm{~m}$ in [4] and 8 , 3 and 2 minutes in [6]) which is chosen to maximize the difference between traffic variables under anomalous conditions and those under normal conditions. We have shown in $[1,9]$ that the effectiveness of an anomaly detection algorithm of this type is highly dependent on the choice of the size of its time window. That is, longer time windows are needed to capture changes under lowflow high-speed conditions and vice versa. Therefore to be deployable, an anomaly detection algorithm based on microscopic traffic variables, needs to be able to identify different types of traffic anomalies under different traffic conditions.

In this paper we propose a novel anomaly detection and classification algorithm that uses microscopic traffic variables and exhibits significant performance improvement over the algorithms presented in [1]. Unlike other previously reported approaches $([4,6])$ where a particular interval prior to incidents has to be selected in order to calibrate the models, the proposed algorithm can be trained over different ranges of intervals prior to incidents and can then be deployed to monitor different time periods of the day to detect traffic anomalies. We also assess the potential of deploying the algorithm in $\mathrm{V} 2 \mathrm{~V}$ and V2I environments where only a fraction of individual vehicle information might be available at a given time due to limitations in the transmission mediums and/or loss of data due to transmission delays [10].

The paper is organized as follows: a review of related studies is provided in Section II. Section III describes the assessment framework, and the proposed detection methodology is presented in Section IV. In Section V, we use real-world data to demonstrate the effectiveness of our proposed algorithm in the detection and classification of anomalies, and the final remarks and future work are outlined in Section VI.

\section{RELATED WORK}

A. Anomaly detection and classification using macroscopic traffic variables

Macroscopic traffic variables representing aggregated behaviour of vehicles are derived using an analogy with fluid dynamics and the relationships between these variables are described in the classical Lighthill-WhithamRichards model [11]. Basic macroscopic traffic variables, derived from inductive loop detectors, notably flow and occupancy, have been extensively employed for traffic incident detection focusing on detection after a major disruption of traffic has occurred [7, 12]. Recently, a number of studies have suggested that it is worth attempting to detect traffic anomalies prior to the occurrence of an incident [4-6]. The majority of these studies have shown that measurements of speed deviation can be used as precursor signals of a traffic incident. Unfortunately, one of the main problems of all these approaches is that measuring variation of speeds at a specific location lacks the microscopiclevel characteristics that could capture individual vehicle interactions over time; the spatial-temporal microscopic characteristics will be lost once the vehicle passes the detector location.

\section{B. Anomaly detection and classification using micro- scopic traffic variables}

Microscopic traffic variables describe individual vehicle behaviours as well as their interactions, and are known to provide fine-grained information about individual vehicles $([11,13])$. However, few studies have employed microscopic traffic variables for anomaly detection. The study in [14] is among the first to use relative speed and inter-vehicle spacing to derive a reliability model for freeway traffic flow, but the model itself is used with macroscopic traffic variables, e.g. flow and density, and no algorithm is suggested for anomaly detection.

Relative speed, inter-vehicle spacing, inter-vehicle time gap and lane change tracking are microscopic traffic variables that have been used for anomaly detection [1517]. In [15], lane changing fractions estimated from loop detectors are used for incident detection, but the effectiveness of this approach depends on the loop detector locations. In VGrid [16], each vehicle only uses its local information to determine whether it is in a queue and if so, communicate this information to other vehicles. A more recent system called WILLWARN uses on-board sensors to measure microscopic information (e.g. wheel speed, reduced friction) to detect possible hazards [18]. 
However, the information shared among vehicles is normaly restricted to hazard-warning messages. Both VGrid and WILLWARN are not explicitly assessed in terms of FAR as to whether they can effectively use shared microscopic traffic variables for anomaly detection and classification.

Recently proposed anomaly detection systems, namely VII-SVM, VII-ANN [19] and NOTICE [17] have been designed to use speed profile and lane changing behaviour of individual vehicles. However, in order to obtain such fine-grained information, these systems require a specific infrastructure that consists of sensors and wireless transceivers installed uniformly on each road segment [17,19] and/or on each lane [17]. Such requirements are likely to limit the deployment of VIISVM, VII-ANN and NOTICE as they are not scalable with typical traffic monitoring systems.

We have recently proposed two algorithms that use the variability of microscopic traffic variables from V2V and V2I communications for anomaly detection even when the information is available only from a fraction of vehicles on the segment [1,9]. In [9] the algorithm uses relative speed and lane changing trajectories to detect anomalies caused by blocked lanes, this algorithm outperforms the algorithm in [15] under low vehicle density. We have also shown that the algorithm in [1] can detect anomalies in a real-world data set and outperform the algorithms in $[4,15]$, but its performance misses certain anomalies that occur under high vehicle density.

\section{ANALysis FramewORK}

We here introduce a distributed traffic monitoring framework that can be supported by the information shared among neighbouring vehicles. This information would enable the calculation of microscopic traffic variables statistics to detect and classify anomalies on a short road segment (e.g. 100-200 meters [20]) and within a short time interval (e.g. 5-30 m [21]). The relevant information (e.g. instantaneous speed and position) would be measured by vehicles sharing information through automotive navigation systems and wireless communications [16,22]. Alternatively, the microscopic traffic information could be inferred from currently available road-side infrastructure, e.g., video CCTV surveillance cameras [23].

In the proposed framework, an anomaly detection model $H=\{F, C, D\}$ is stored and can be activated locally when vehicles are present on the segment of interest. $H$ generally consists of three entities: $F$ denotes a feature extraction model, $C$ denotes a classification model and $D$ denotes a decision-making model, that

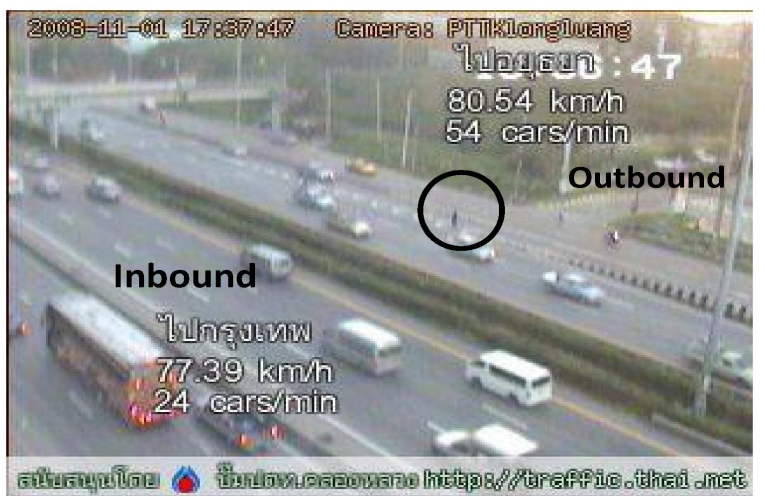

Fig. 1: Transient anomaly caused by a pedestrian on the shoulder of a freeway segment.

have been trained with historical data. The parameters of model $H$ can be downloaded by the vehicles on the segment or used by a local roadside infrastructure itself for anomaly detection. The input to the model consist of the microscopic traffic variables that can be calculated and shared amongst individual vehicle and the closest vehicle(s) downstream whose information is available at the time of interest.

In this study, we consider two types of anomalies, 1) Transient Anomalies and 2) Disruption Precursors. Transient Anomalies are defined as deviations of traffic patterns that may be followed by minor disruptions to the traffic flow. An example of a transient anomaly is shown in Fig. 1 where an unexpected appearance of a pedestrian on the freeway shoulder causes distraction and a temporary drop in speed. Disruption Precursors are defined as traffic patterns that may lead to a major disruption of the underlying traffic flow. This type of anomalies has received much attention primarily in cases where it is associated with accidents [4], crashes [6], or congestions $[5,7]$. Figure 2 shows an incident that has evolved from a lane-blocking and is disrupting traffic flow. The aim in this paper is to develop an algorithm that can identify the onset of both types of traffic anomaly as the first step towards detecting, classifying and predicting the impact of the incident so that appropriate, proactive, neutralizing and coordinated set of actions can be initiated.

\section{Methodology}

\section{A. Overview of the proposed algorithm}

The proposed algorithm is based on the observation that a traffic anomaly can be detected by monitoring the changes in the behaviour of individual vehicles (e.g. deceleration and lane change), that would be reflected in changes in the variability of the observed microscopic 


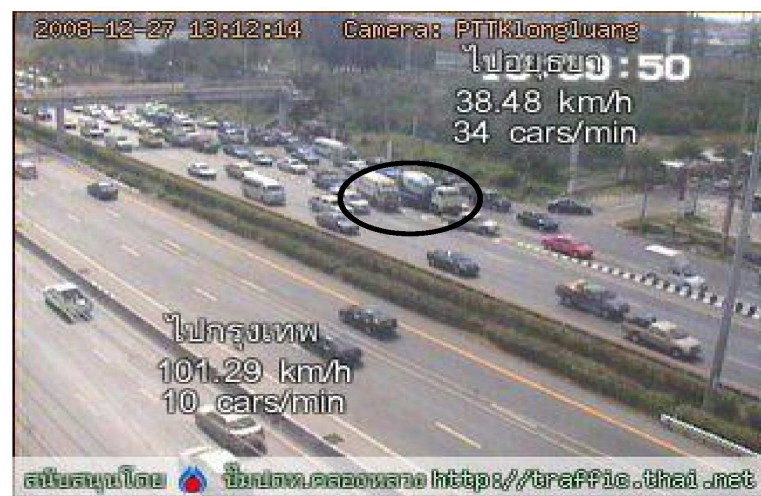

Fig. 2: Disruption precursor caused by a truck that blocked a lane.

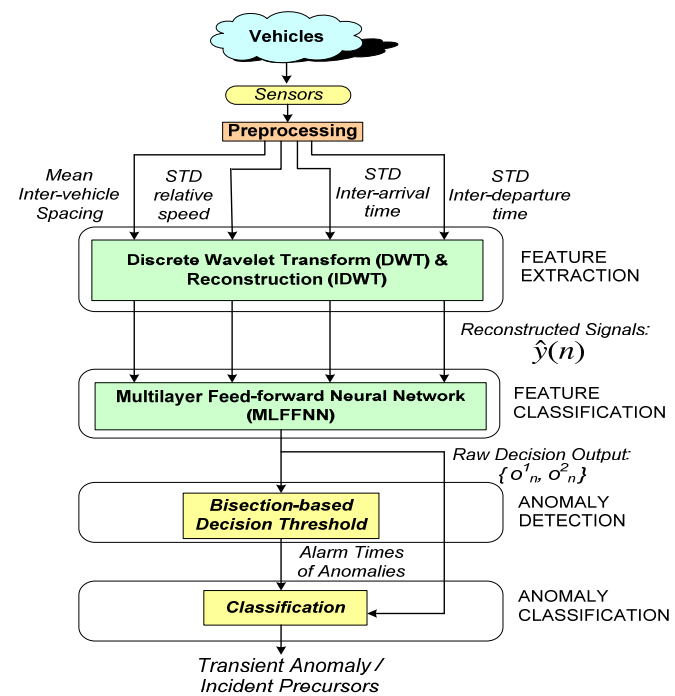

Fig. 3: The proposed anomaly detection and classification methodology.

traffic variables [1]. More recently it has also been suggested that microscopic traffic variables analysis could be used to identify incident precursor phases [8].

The proposed anomaly detection scheme can be divided into major blocks or stages as depicted in Fig.3. The process starts with the feature extraction stage which performs the conversion of the original traffic variables into features that are abstract representation of those variables, and which contain all the essential information for the detection task. In this work, the feature extraction step is based on the use of a wavelet-based filter. For that purpose, each variable is initially decomposed into low frequency and high frequency components. Then, the small coefficients of the high frequency components that are less correlated with the original microscopic signals are removed, and finally the filtered signal is reconstructed. In the next block, referred to as feature classification, every new measurement of the microscopic variables is assigned to a specific class according to the characteristics of the reconstructed filtered signals. In this paper a Multi-layer Feed Forward Neural Network (MLFFNN) is selected for the feature classification model on the basis of previous performance [24,25]. The reconstructed time series of microscopic statistics are then used as inputs for the MLFFNN. The outputs of MLFFNN represent degrees of likelihood between 0 and 1 and hence a threshold is needed to discover and classify an emerging anomaly pattern. Here we use a bisectionbased decision threshold [26] which is optimized over different traffic conditions to enhance the adaptability of the proposed algorithm. An alarm is raised if the MLFFNN outputs exceed the bisection-based decision threshold for a certain number of consecutive samples (persistency (PT)). Finally, the alarm times and the outputs from MLFFNN are used to classify the detected traffic anomalies.

\section{B. Inputs description}

The microscopic traffic variables used in this study for anomaly detection are 1) inter-vehicle spacing: $s_{i, n}=$ $x_{i-1, n}-x_{i, n}$ where $x_{i, n}$ is the position of a vehicle $i$ at time $n$,2) relative speed: $v_{i, n}=w_{i-1, n}-w_{i, n}$ where $w_{i, n}$ is the speed of a vehicle $i$ at time $n, 3$ ) inter-arrival time $a_{i}$ and 4) inter-departure time $d_{i}$. The inter-arrival time is defined as the difference between the arrival time to the beginning of a segment of interest of a vehicle $i$ and that of the previous vehicle $i-1$ that has arrived. Similarly, the inter-departure time is defined as the difference between the arrival time to the end of a segment of interest of a vehicle $i$ and the arrival time of the previous vehicle $i-1$.

We select as statistics of interest the standard deviations of relative speed, inter-arrival time and interdeparture time of vehicles to track changes in variability. To further enhance the learning capabilities of the algorithm under different traffic conditions and with different vehicle density, we select the mean of intervehicle spacing to track changes in vehicle density [27].

\section{Feature extraction}

The effectiveness of the anomaly detection algorithm is first and foremost determined by the representativeness of the features and how efficiently they can be extracted and identified. Our analysis with a real-world data set in [1] has shown that some anomalies may 
have weak amplitudes and in some cases the boundary between normal and anomalous behaviour cannot be clearly distinguished. Also, changes associated with anomalies can occur under different time scales depending on the underlying traffic conditions. As we would intend to deploy the algorithm in nodes that might have limited storage and communications capabilities the feature extraction model has to be minimal. Hence, the process of feature extraction is performed by the use of wavelet transforms, which is a well-known technique for the analysis of signals with multi-scale behaviour [2]. Discrete Wavelet Transform (DWT) is chosen as it can represent information signals with orthogonal wavelet basis which minimize the number of associated representative coefficients [28]. Furthermore, DWT can represent the original signal using wavelet detail coefficients (fine-scale information) and approximation coefficients (coarse-scale information), which would enhance the ability to extract changes in microscopic traffic variables associated with different time scales. In the proposed scheme, DWT is used to individually filter relevant components of the four input microscopic traffic variables described in Section IV-B. The output of the filtering process is the reconstruction of each of these microscopic traffic variables using only the approximation coefficients and the wavelet coefficients that are highly correlated with the original signal.

Based on DWT framework, a signal $y(n)$ is decomposed into levels of approximations and details to form a multi-resolution analysis of the signal as: $y(n)=\sum_{k} a_{J, k} \phi_{J, k}(n)+\sum_{m>J} \sum_{k} d_{m, k} \psi_{m, k}(n)$ where $J, m, k \in \mathbb{Z}, a_{J, k}$ denotes the approximation coefficient at resolution $J, d_{m, k}$ denotes the wavelet coefficient at resolution $m, \phi_{J, k}(n)$ is a scaling function and $\psi_{m, k}(n)$ is a wavelet function at resolution $m$. The coefficients $a_{L, k}$ and $d_{m, k}$ are computed as: $a_{J, k}=$ $\left\langle y(n), \phi_{J, k}(n)\right\rangle, d_{m, k}=\left\langle y(n), \psi_{m, k}(n)\right\rangle \quad m, k \in \mathbb{Z}$, where the operator $\langle$.$\rangle denotes the inner product in$ the space of square integrable functions $J^{2}(\mathbb{R})$. The dyadic DWT assumes scaling functions $\phi$ and wavelet functions $\psi$ of the form: $\phi_{m, k}(n)=2^{m / 2} \phi\left(2^{m} n-k\right)$, $\psi_{m, k}(n)=2^{m / 2} \psi\left(2^{m} n-k\right) \quad m, k \in \mathbb{Z}$. Particularly, DWT is used to obtain the approximation coefficients $a_{L, k}$ and wavelet coefficients $d_{m, k}$ while the process of reconstructing the signal given such coefficients is named the Inverse Discrete Wavelet Transform (IDWT). In practice, the dyadic DWT can be implemented in a computationally efficient manner using the dyadic filter tree algorithm [28].

\section{Feature classification}

Identifying anomalies can be a difficult task due to possible non-linear relationships between patterns of microscopic traffic variables and classes (normal, transient anomaly and disruption precursors) [1]. These relationships could become even more complex when there is a change in the underlying traffic condition, because a sample of microscopic traffic variables associated with one class in one traffic condition may belong to another class in another traffic condition. In order to capture such non-linear relationships a Multi-layer Feed-Forward Neural Network (MLFFNN) is selected for feature classification because once properly trained, MLFFNN has often been found to perform well in classifying traffic patterns when deployed on site [24, 25, 29].

For a classification problem, the primary task of MLFFNN is to learn a classification function: $C: \underline{Y} \rightarrow$ $\underline{O}$, where $\underline{Y}=\left\{y_{n}^{i}\right\}$ is a set of input to the $i^{t h}$ node in the input layer of MLFFNN, and $\underline{O}=\left\{o_{n}^{k}\right\}$ is an output vector from the $k^{t h}$ node in the output layer at time $n$ [30]. Let $w^{i j}$ denote the weight for the connection from node $i$ in the input layer to node $j$ in the hidden layer, and $v^{j k}$ denote the weight for the connection from node $j$ in the hidden layer to node $k$ in the output layer. A sigmoid function, $S I G(x)=\frac{1}{1+e^{-x}}$, is used as a transfer function in the nodes in both the hidden and output layers, which can be represented as :

$$
\begin{aligned}
& z_{n}^{j}=S I G\left(\sum_{i} w^{i j} y_{n}^{i}\right) . \\
& o_{n}^{k}=S I G\left(\sum_{j} v^{j k} z_{n}^{j}\right) .
\end{aligned}
$$

Based on preliminary assessment of the algorithm with the training data set, MLFFNN with two hidden layers is chosen for feature classification. Let $m_{0}, m_{1}, m_{2}$ and $m_{3}$ denote the number of neurons in the input layer, the first hidden layer, the second hidden layer and the output layer of the proposed algorithm respectively. As there are four microscopic traffic variables for input, the input layer consists of four neurons, i.e. $m_{0}=4$. The number of neurons in each hidden layer is chosen as a function of the number of microscopic traffic variable inputs with the aim of balancing between not having too few neurons (e.g. the capability to model non-linear mapping) and not having too many neurons (e.g. the problems of excessive time-consuming and having too many local minimums). We choose the same set up as in [25,31] where $m_{1}=m_{2}=3 * m_{0}+1$. Each element in the output of the MLFFNN, $\left\{o_{n}^{1}, o_{n}^{2}\right\}$, is set to be between 0 and 1 which would require a decision-making 
threshold to classify whether the output corresponds to an anomaly.

\section{E. Decision algorithm for anomaly detection}

It is crucial that during the training process, the proposed algorithm can examine a range of possible output values to find an optimal decision-making threshold for assessing the outputs of MLFFNN. We employ a threshold varying bisection method in [26] which can be summarized as follows. During a training process, a range of threshold $[\alpha, \beta], 0 \leq \alpha \leq \beta \leq 1$, is considered where the goal is to find a threshold $\gamma$ that minimizes the cost function $f_{\gamma}$. At each step, $f_{\alpha}, f_{\beta}$ and $f_{\frac{\alpha+\beta}{2}}$ are compared. If $f_{\frac{\alpha+\beta}{\beta^{2}}}<\min \left(f_{\alpha}, f_{\beta}\right)$ then the algorithm selects $\gamma=\frac{\alpha+\beta^{2}}{2}$ and exits, otherwise it repeats the previous step but with a bisected threshold range $\left[\frac{\alpha+\beta}{2}, \beta\right]$ if $f_{\alpha}>f_{\beta}$ or $\left[\alpha, \frac{\alpha+\beta}{2}\right]$ if $f_{\alpha} \leq f_{\beta}$. The bisection process is repeated until the minimum cost function is reached, i.e. $f_{\gamma}<\min \left(f_{\alpha}, f_{\beta}\right)$, or the current lower bound is at least $\epsilon$ less than the current upper bound, i.e. $\beta-\alpha \leq \epsilon$. In the latter case, the algorithm selects $\gamma=\beta-\epsilon$ as its final threshold, which is used to assess the raw decision output from MLFFNN. In the proposed scheme, a lower bound of $\alpha=0.3$ and an upper bound of $\beta=0.7$ are chosen because a larger initial interval (e.g. [0.1,0.9]) could cause the bisection method to select the threshold $\gamma$ that is too biased toward a training set dominated by a particular traffic condition (e.g. too close to 0.1 or 0.9 ) [26].

To enable the decision algorithm to select a threshold $\gamma$ that maximize DR and minimize FAR, we use the cost function $f_{\gamma}=w \times F A R_{\gamma}+(1-w) \times\left(1-D R_{\gamma}\right)$ for the modified bisection, where $F A R_{\gamma}=$ false alarm rate and $D R_{\gamma}=$ detection rate when a threshold $\gamma$ is used. Hence, the term $1-D R_{\gamma}$ corresponds to the number of missed detections. The choice of $w(0 \leq w \leq 1)$ depends on whether maximizing DR or minimizing FAR is more important which subsequently depends on practical considerations and the purpose of the observations. In this experiment, the value of 0.5 is chosen for $w$ to balance between maximizing DR and minimizing FAR.

The raw decision output from MLFFNN shown in Fig. 3 consists of two separate signals, $\left\{o_{n}^{1}, o_{n}^{2}\right\}$, whose binary combination, at time $n$, determines whether an anomaly has occurred as well as the type of the detected anomaly; $\{0,0\}$ for normal traffic patterns, $\{0,1\}$ for transient anomalies and $\{1,1\}$ for disruption precursors. For the anomaly detection decision, persistency (PT) is used to determine the number of consecutive samples of the raw decision output, $\left\{o_{n}^{1}, o_{n}^{2}\right\}$, that exceed the threshold. The alarm is raised if at least one element of $\left\{o_{n}^{1}, o_{n}^{2}\right\}$ exceeds the threshold consecutively for a number of PT samples.

\section{F. Decision algorithm for anomaly classification}

The classification decision function is based on the assessment of the likelihood that an anomaly corresponds to a transient anomaly or a disruption precursor. In the proposed algorithm, the assessment of likelihood is performed on each raw decision output of the MLFFNN, which is modelled as a classifier whose value represents likelihood [32]. The likelihood combination techniques in [32] are originally proposed to combine outputs over space from different NNs. In this paper, we apply the same concept to combine the raw decision output over time from the same $\mathrm{NN}$, i.e. for a given time $n$, the raw decision output $\left\{o_{1}, o_{2}\right\}_{n}$ is itself a classifier. The classification decision function $f(\eta)$ can be formalized as shown in (3), where the raw decision outputs of MLFFNN are monitored from the time of detection $n_{0}$ to $n_{0}+\eta$, where $\eta$ denotes a classification interval, i.e. $\left\{o_{1}, o_{2}\right\}_{n_{0}},\left\{o_{1}, o_{2}\right\}_{n_{0}+1}, \ldots,\left\{o_{1}, o_{2}\right\}_{n_{0}+\eta}$.

$$
f(\eta)=\underset{c}{\arg \max } \sum_{n=n_{0}}^{n_{0}+\eta} w_{n}^{c} f_{n}^{c}, c=1,2 .
$$

The weight $w_{n}^{c}$ is the weighted average of the likelihood $f_{n}^{c}$, i.e. $w_{n}^{c}=\frac{f_{n}^{c}}{\sum_{c=1,2} f_{n}^{c}}$. The likelihood $f_{n}^{c}$ is calculated from the raw decision outputs $\left\{o_{n}^{1}, o_{n}^{2}\right\}$. Recall that the raw decision output $o_{n}^{1}$ nearer to zero indicates more likelihood of transient anomaly while the raw output $o_{n}^{1}$ closer to one indicates more likelihood of disruption precursors. Hence, $f_{n}^{c}$ is calculated as: $f_{n}^{1}=\left(1-o_{n}^{1}\right) \times o_{n}^{2}$ and $f_{n}^{2}=o_{n}^{1} \times o_{n}^{2}$.

\section{G. Distributed deployment of the proposed algorithm}

The proposed algorithm is designed such that the anomaly detection and classification functions are performed in a distributed manner by neighbouring vehicles and/or by a local road-side infrastructure, based on the locally collected information. Figure 4 shows an example of how microscopic traffic information of individual vehicles can be used by the proposed algorithm to detect traffic anomalies on the road segment of interest. In this example, the proposed algorithm is used by vehicle 7 to identify traffic anomalies based on microscopic traffic information from downstream vehicles 1-6. In this setting each vehicle learns microscopic traffic variables of other vehicles by exchanging information about itself with other vehicles in the neighbourhood. In the figure, vehicle 6 collects and sends to vehicle 7 microscopic 


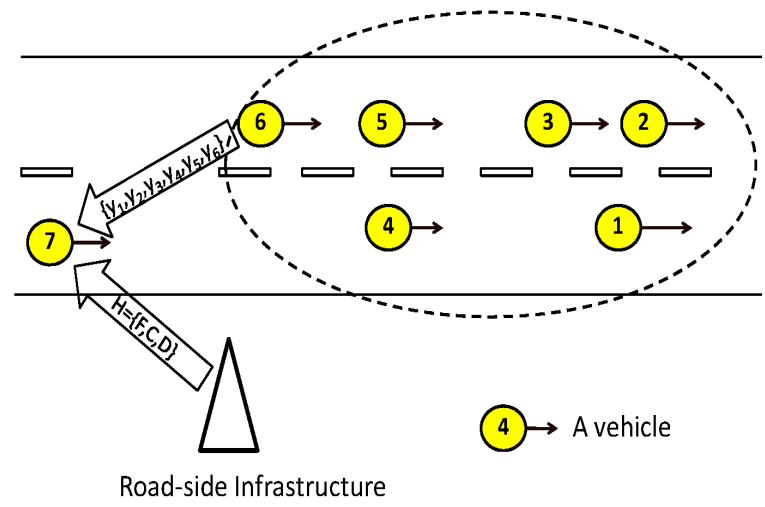

Fig. 4: An example of distributed deployment of the proposed algorithm.

traffic variables $\left\{y_{i}, i=1, . ., 6\right\}$ (speeds, positions, arrival and departure times) of other vehicles on the segment where the mean of inter-vehicle spacing and the standard deviations of relative speed, inter-arrival time and inter-departure time are calculated based on the received microscopic information. The anomaly detection and classification model $H$ for the road segment is downloaded by vehicle 7 from, e.g. a road-side infrastructure.

\section{Performance Evaluation using REAL-WORLD DATA}

\section{A. Performance evaluation parameters}

Let $n_{p, q}$ be the time that the $p^{\text {th }}$ alarm is raised for an anomaly recorded at a time point $n_{q}$. The alarm is considered a true alarm if $n_{q, p} \in\left[n_{q}, n_{q}+n_{b}\right]$, otherwise it is considered a false alarm, in which case $n_{b}$ denotes a detection interval used for evaluation purpose only and should not exceed anomaly duration. Given $M$ anomaly cases in the experiment, the performance evaluation parameters we consider are Detection Rate $(D R)$, Mean Time to Detection (MTTD) and False Alarm Rate $(F A R)$ :

$$
\begin{gathered}
D R=\frac{\text { Number of anomalies detected }}{M} . \\
M T T D=\frac{\sum_{p=1}^{M}\left(n_{q, p}-n_{q}\right)}{M}, n_{q, p} \in\left[n_{q}, n_{q}+n_{b}\right] . \\
F A R=\frac{\text { Number of Alarms not in }\left[n_{q}, n_{q}+n_{b}\right]}{\text { Total number of Alarms }} .
\end{gathered}
$$

\section{B. Descriptions of the data}

In order to assess the algorithm, we chose a realworld freeway segment in which the microscopic traffic variables can also be obtained from a video surveillance camera (see Figures 1 and 2). The road segment we studied is part of a freeway network that links Bangkok to the Northern provinces of Thailand. During the 5month period of data collection, the free-flow maximum and average speeds on this freeway segment were found to be $120 \mathrm{~km} /$ hour and $80 \mathrm{~km} /$ hour respectively. The nearest junctions are approximately $1.2 \mathrm{~km}$ upstream and $1.5 \mathrm{~km}$ downstream. Recurring traffic congestion occurs during morning rush hours between $7 \mathrm{am}$ to $9 \mathrm{am}$, where congestion usually originates at the downstream junction and propagates upstream to the freeway segment studied during these periods. We note that these recurring congestions are not included as anomaly cases in this paper.

1) Calculation of traffic variables: The image processing software used on this camera to track individual vehicles has been developed by a team of researchers and is reported in [23]. The object tracking accuracy of this camera has been estimated to be approximately $80 \%$. Even though the proposed algorithm was initially assessed using data from video camera, it can also be deployed with the support of a VANET based information sharing system in which individual vehicle tracking is performed by automatic vehicle location systems (e.g. GPS), real-time location systems (e.g. RFID) or inertial navigation systems (e.g. dead reckoning) and the information can be shared using wireless communication technologies such as cellular GPRS, radio wave or infrared $[16,22,33]$. A recent study in a simulated environment reported in [34] has shown that vehicular speed can be estimated using on-board vehicle data with error as low as $10.5 \%$.

On the image frame of the camera as shown in e.g Fig. 2, virtual entrance and exit lines were drawn at the beginning and the end of the segment respectively. For a vehicle $i,\left\{t_{i}^{i n}, t_{i}^{\text {out }}, w_{i}^{\text {emp }}\right\}$ was recorded, where $t_{i}^{i n}$ is the time that the vehicle crossed the entrance line, $t^{\text {out }}$ is the time that the vehicle crossed the exit line, and $w_{i}^{\text {emp }}$ is speed of vehicle $i$ calculated as $w_{i}^{\text {emp }}=\frac{\text { Segment Length }}{t^{\text {out }}-t_{i}^{\text {in }}}$. We note that the accuracy of individual vehicle speed will not have significant impact on the performance of the proposed algorithm as it does not rely on measurements of individual vehicle to detect anomalies (see Section IV-B).

The inter-arrival time is calculated as $a_{i}^{\text {emp }}=t_{i}^{i n}-$ $t_{i-1}^{i n}$, while the inter-departure time is calculated as $d_{i}^{\text {emp }}=t_{i}^{\text {out }}-t_{i-1}^{\text {out }}$. For a sampling interval of $\tau$ seconds, the averages of inter-arrival time $\left(\overline{a_{m \tau}^{\mathrm{emp}}}\right)$ and inter-departure time $\left(\overline{d_{m \tau}^{\mathrm{emp}}}\right)$ and the standard deviations 
of inter-arrival time $\left(\sigma_{a, m \tau}^{\mathrm{emp}}\right)$ and inter-departure time $\left(\sigma_{d, m \tau}^{\mathrm{emp}}\right)$ of the sampling interval $m \tau$ can be calculated as: $\overline{a_{m \tau}^{\mathrm{emp}}}=\frac{\sum_{i=1}^{I} a_{i}^{\mathrm{emp}}}{I}, \sigma_{a, m \tau}^{\mathrm{emp}}=\sqrt{\frac{\sum_{i=1}^{I}\left(\left(a_{i}^{\mathrm{emp}}\right)^{2}-\left(\overline{a_{m \tau}^{\mathrm{emp}}}\right)^{2}\right)^{2}}{I}}$ for $(m-1) \tau \leq t_{i}^{i n}<m \tau, \overline{d_{m \tau}^{\mathrm{emp}}}=\frac{\sum_{i=1}^{I} d_{i}^{\mathrm{emp}}}{I}, \sigma_{d, m \tau}^{\mathrm{emp}}=$ $\sqrt{\frac{\sum_{i=1}^{I}\left(\left(d_{i}^{\mathrm{emp}}\right)^{2}-\left(\overline{d_{m \tau}^{\mathrm{emp}}}\right)^{2}\right)^{2}}{I}}$ for $(m-1) \tau \leq t_{i}^{\text {out }}<m \tau$ where $m=1,2,3, \ldots$

Since the camera is not yet capable of directly measuring spaces between individual vehicles, the average inter-vehicle spacing is instead calculated from the average inter-departure time and average speed as: $\overline{s_{m \tau}^{\mathrm{emp}}}=\overline{d_{m \tau}^{\mathrm{emp}}} \times \overline{w_{m \tau}^{\mathrm{emp}}}$, where $\overline{w_{m \tau}^{\mathrm{emp}}}=\frac{\sum_{i=1}^{I} w_{i}^{\mathrm{emp}}}{I}$. A relative speed observed by vehicle $i$ to its leading vehicle $i-1$ is calculated as $v_{i}^{\text {emp }}=w_{i-1}^{\text {emp }}-w_{i}^{\text {emp }}$ for $t_{i}^{\text {out }}>t_{i-1}^{\text {out }}$. Then, for a sampling interval of $\tau$ seconds, the average $\left(\overline{v_{m \tau}^{\mathrm{emp}}}\right)$ and standard deviation $\left(\sigma_{m \tau}^{\mathrm{emp}}\right)$ of relative speeds of the sampling interval $m \tau$ can be calculated as: $\overline{v_{m \tau}^{\mathrm{emp}}}=\frac{\sum_{i=1}^{I} v_{i}^{\mathrm{emp}}}{I}$ and $\sigma_{m \tau}^{\mathrm{emp}}=\sqrt{\frac{\sum_{i=1}^{I}\left(\left(v_{i}^{\mathrm{emp}}\right)^{2}-\left(\overline{v_{m \tau}^{\mathrm{emp}}}\right)^{2}\right)^{2}}{I}}$ for $(m-1) \tau \leq t_{i}^{\text {out }}<m \tau$, where $m=1,2,3, \ldots$

2) Obtaining anomaly cases: The real-world data set used in this paper was collected by the camera under different traffic conditions daily from 6am to $6 \mathrm{pm}$ over a five month period from August to December 2008, plus an additional two months of video post-processing to visually identify anomaly cases. The period of 5 months obtained sufficient anomaly cases for performance evaluation. The average number of vehicles detected passing the freeway segment is approximately 30,000 vehicles per day so there are approximately 5,000,000 records for the 5-month period of data collection. For evaluation purposes, anomalies that took place were independently logged by a team of transportation researchers using video images from the camera at the target site. The video images were used to log anomalies identified as disruption precursor when they were associated to major traffic disruption, otherwise the anomaly were recorded as transient anomalies. There are 26 anomaly cases in the data, nine cases were transient anomalies and 17 were disruption precursors. These anomalies took place under various traffic conditions, such as low-flow high-speed, high-flow high-speed and low-flow low-speed.

Based on video images, transportation researchers were also asked to log the start and end times of the anomaly cases. Each anomaly case consists of a set of timestamps $\left\{T_{s}^{a}, T_{s}^{i}, T_{e}^{i}\right\}$, where $T_{s}^{a}$ denotes the time when traffic anomaly is recorded (e.g. a pedestrian and a lane-blocking truck are observed in Figures 1 and 2 respectively), $T_{s}^{i}$ denotes the times when a traffic incident is recorded to take place and $T_{e}^{i}$ denotes the end time of traffic incident or when traffic has recovered. For transient anomalies, only $T_{s}^{a}$ and $T_{e}^{i}$ are recorded as incident occurrence is not observed. The duration of traffic anomalies analyzed in this paper ranges from 5 to 45 minutes. In the performance evaluations in Section V-D and V-E, an anomaly is considered detected if an alarm is raised by the algorithm within $\left[T_{s}^{a}, T_{s}^{i}\right]$ for disruption precursors and $\left[T_{s}^{a}, T_{e}^{i}\right]$ for transient anomalies. Then, the MTTD is calculated as the average of the difference between the alarm time and $T_{s}^{a}$.

\section{Benchmark anomaly detection and classification al- gorithm [1]}

We chose the algorithm proposed in [1] as our benchmark, since it has been shown to outperform algorithms in $[4,15]$. The benchmark algorithm combines decision regarding temporal changes in the variances of microscopic variables such as, relative speed, inter-arrival and inter-departure of vehicles, with spatial changes related to upstream and downstream traffic patterns. An alarm was activated using a weighted vote scheme.

\section{Anomaly detection assessment}

The experiment reported in this section is conducted using a cross-validation technique. Anomaly cases in our data were separated into disjoint training and testing sets; the training set consists of 12 anomaly cases and the testing set consists of 14 anomaly cases. The training set was used to train the MLFFNN and to find the optimal threshold by the bisection method in the proposed algorithm, while the testing set was used for performance evaluations. In each experiment, the anomaly cases in the training and testing sets were selected randomly from the 26 anomaly cases in our data set. Furthermore, in order to reflect real-world operational conditions, each algorithm was assessed continuously from 6 am to $6 \mathrm{pm}$ and for each day of the data set.

Table I shows performance evaluation results DR, FAR and the MTTD, obtained by averaging over 20 experiments. The detection interval $n_{b}$ of each anomaly case in equations (4), (5), and (6) is the recorded duration of that anomaly case which is found to be between 5 and 45 minutes.

The proposed algorithm detects a relatively higher number of anomalies with much smaller FAR than the benchmark algorithm. Furthermore, the proposed algorithm detects anomalies that are missed in [1]. We note that the missed anomaly cases took place while there was already a high flow of vehicles ( $\geq 2000$ vehicles/hour) on the segment and where the boundary between the variability of relative speed associated with normal and 
TABLE I: Performance comparisons results obtained by averaging 20 experiments for different values of persistency PT

\begin{tabular}{|c|c|c|c|}
\hline Proposed Algorithm (Figure 3) & DR & $F A R$ & $M T T D$ \\
\hline PT $=1$ & 1.00 & 0.057 & 469.9 \\
PT $=2$ & 1.00 & 0.050 & 516.3 \\
PT = & 0.95 & 0.035 & 516.4 \\
\hline Benchmark Algorithm [1] & $D R$ & $F A R$ & $M T T D$ \\
\hline PT =1 & 0.88 & 0.370 & 370.4 \\
PT = & 0.73 & 0.170 & 139.1 \\
PT = 3 & 0.69 & 0.150 & 373.1 \\
\hline
\end{tabular}

anomalous traffic conditions cannot be clearly distinguished.

The proposed algorithm successfully classifies anomaly cases that cannot be detected by the benchmark algorithm [1], and this is reflected in the slightly higher MTTD values shown in Table I. These anomalies are associated with a high volume of vehicles on the freeway segment where the variability of the microscopic variables is bounded by the lack of room to maneuver vehicles. Consequently, it takes longer for the proposed algorithm to detect subtle changes in microscopic variability. Furthermore, we note that MTTDs shown in Table I are the average detection delays of traffic anomalies, not the average detection delays of traffic incidents. All the anomalies included in the test data set are detected before the actual (independently recorded) traffic incidents time are logged and/or before the observed traffic patterns settled back to e.g. normal free-flow conditions. Further analysis on these real-world data sets shows that the alarm times of the proposed algorithm are on average triggered 13 minutes before the occurrence of incidents which should still give enough time to initiate an appropriate response.

\section{E. Anomaly detection with partial coverage of vehicle information}

1) Anomaly detection with partial availability of data from vehicle population: The statistics of microscopic traffic variables are calculated from a percentage of the vehicles population $I=P o A * I_{\text {total }}$, where $P o A$ is the Percentage of Availability and $I_{\text {total }}$ is the total number of vehicles on the segment. Figure 5 shows DR and FAR when the proposed algorithm have access to $P o A$ ranges from $10 \%$ to $100 \%$. The proposed algorithm can detect all anomaly cases with FAR less than $20 \%$ for $50 \% \leq$ $P o A \leq 100 \%$. As the average vehicle density $I_{\text {total }}$ was observed to be between 6-20 vehicles/lane, the results in Fig. 5 shows that the proposed algorithm can still identify anomalies in the real-world data set even when

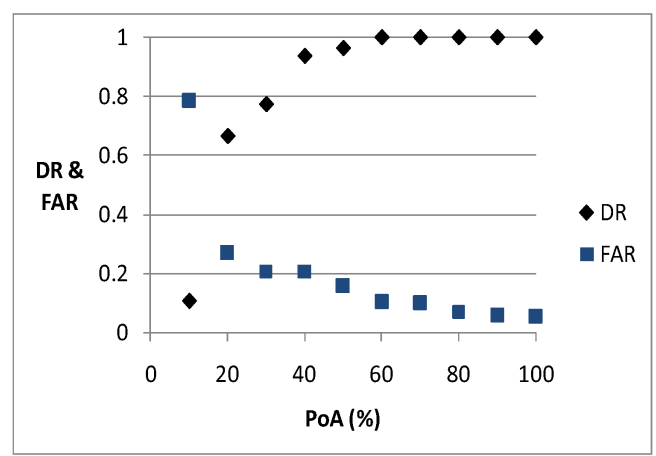

Fig. 5: Detection rates and false alarm rates under different percentage of availability (PoA)

the microscopic traffic information is available only for $50 \%$ of vehicles on the segment, (i.e. for $I$ as low as 3 vehicles/lane). As $P o A$ is further reduced to the range $\leq$ $40 \%$ (i.e. $I \approx 2-3$ vehicles/lane) the information is no longer sufficient to identify all traffic anomalies. Hence, in order to keep the DR and FAR at acceptable levels, the proposed algorithm has to be trained particularly for low $P o A$ with sufficient number of anomaly cases.

2) Anomaly detection with aggregated information: In this experiment, a ratio-based aggregation algorithm adopt from TrafficView system [10] is employed to form clusters of vehicles where microscopic traffic variables of individual vehicles are locally aggregated according to the availability of Medium Access Control (MAC) payload size, the amount of microscopic traffic information, and the relative distances of vehicles with respect to one another.

In the ratio-based algorithm, a cluster is formed where every vehicle makes itself known to other vehicles, and the upstream-most vehicle in the cluster is selected as a cluster-head. The cluster-head determines if the remaining MAC payload size $R$ is enough for dissemination of microscopic traffic variables individually. If the size $R$ is not enough, then local aggregations are needed and sub-clusters are formed according to the relative distance between each vehicle to the upstream cluster-head. An example of how microscopic traffic variables are aggregated when the available MAC payload size is reduced to $0.3 R$ is shown in Fig. 6. Let us assume hypothetically that $0.1 R$ is needed to transmit a microscopic traffic variable of each vehicle so the available bandwidth can accommodate only a single vehicle information from each sub-cluster. In this example, vehicle 6 acts as a cluster-head which collects the aggregated information from the two downstream sub-clusters, vehicles $\{1,2,3\}$ 


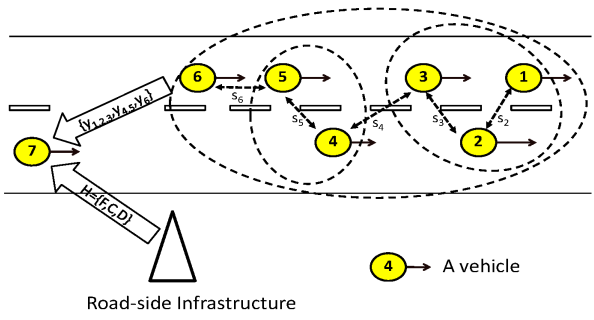

Fig. 6: An example of distributed deployment of the proposed algorithm under information aggregation using e.g. [10].

and vehicles $\{4,5\}$. Let $y_{i}$ denote a microscopic traffic variable measured by vehicle $i$ and $s_{i}$ denotes intervehicle spacing between vehicle $i$ and the closet vehicle downstream. The aggregated microscopic traffic information sent to vehicle 7 would be the weighted average $\left\{y_{1,2,3}, y_{4,5}, y_{6}\right\}$ where $y_{1,2,3}=\frac{\sum_{i=1}^{3} s_{i, 6} y_{i}}{\sum_{i=1}^{3} s_{i, 6}}$, $y_{4,5}=\frac{\sum_{i=4}^{5} s_{i, 6} y_{i}}{\sum_{i=4}^{5} s_{i, 6}}$, and $s_{i, 6}=\sum_{j=1}^{6} s_{j}$. Then, vehicle 7 would use $\left\{y_{1,2,3}, y_{4,5}, y_{6}\right\}$ to calculate the microscopic statistics.

TABLE II: Assessment of the proposed algorithm under different $P o A$ and percentages of MAC payload availability $(R)$ in the real-world data set.

\begin{tabular}{|c|c|c|c|c|c|c|}
\hline \multirow{2}{*}{ PoA (\%) } & \multicolumn{2}{|c|}{ Unlimited $R$} & \multicolumn{2}{|c|}{$0.5 R$} & \multicolumn{2}{c|}{$0.3 R$} \\
\cline { 2 - 7 } & DR & FAR & DR & FAR & DR & FAR \\
\hline 100 & 1.000 & 0.057 & 1.000 & 0.058 & 1.000 & 0.059 \\
90 & 1.000 & 0.058 & 1.000 & 0.059 & 1.000 & 0.064 \\
80 & 1.000 & 0.061 & 1.000 & 0.070 & 1.000 & 0.079 \\
70 & 1.000 & 0.076 & 1.000 & 0.101 & 1.000 & 0.107 \\
60 & 1.000 & 0.104 & 1.000 & 0.104 & 1.000 & 0.161 \\
50 & 1.000 & 0.161 & 0.955 & 0.173 & 0.955 & 0.187 \\
40 & 0.937 & 0.197 & 0.937 & 0.206 & 0.921 & 0.207 \\
30 & 0.767 & 0.197 & 0.774 & 0.206 & 0.671 & 0.228 \\
20 & 0.666 & 0.282 & 0.648 & 0.289 & 0.572 & 0.307 \\
10 & 0.108 & 0.504 & 0.109 & 0.788 & 0.060 & 0.870 \\
\hline
\end{tabular}

Table II shows DR and FAR when the proposed algorithm is applied to detect anomalies in the real-world data set ${ }^{1}$. The MTTD is not shown in this table as it is found to have consistent values of approximately 8-9 minutes for every combination of $P o A$ and $R$ analyzed. The DR and FAR are averaged over 10 experiments where in each experiment, the anomaly cases in the

\footnotetext{
${ }^{1}$ The MAC payload size $R$ is set to be 2313 bytes for an 802.11 bbased wireless network and the average size for a single vehicle record is 50 bytes as in [10].
}

training and testing sets are selected randomly from the 26 anomaly cases in our data set.

Table II shows that the proposed algorithm detects anomalies with more than $90 \%$ DR and FAR less than $20 \%$ even when PoA is as low as $40 \%$ and only $50 \%$ and $30 \%$ of MAC payload ( $0.5 R$ and $0.3 R$ ) are available (information obtained from only 2-3 vehicles). It can also be seen that the reduction of MAC payload size does not have a significant impact on either DR or FAR. However, when $P o A=10 \%$, as previously highlighted, most anomaly cases cannot be detected as microscopic traffic variables are obtained from a single vehicle.

3) Anomaly detection with packet transmission delay: In this experiment, a packet transmission delay is generated from a probability distribution. If the generated packet transmission delay exceeds a time window $L$, the microscopic traffic information of that vehicle is assumed lost. We use not only an Exponential distribution, but as suggested in [35], we also include a generalized Pareto distribution in our tests to generate packet transmission delays.

TABLE III: Assessment of the proposed algorithm on the real-world data set, with packet transmission delays, $P o A=$ $50 \%$ and MAC payload size $=0.5 R$. Time window size is 300s. EXP refers to exponential distribution and GP refers to Generalized Pareto distribution.

\begin{tabular}{|c|c|c|c|}
\hline Packet Transmission Delay (s) & DR & FAR & MTTD(s) \\
\hline No Delay & 0.955 & 0.173 & 448.2 \\
\hline EXP with mean 202.9s & 0.929 & 0.199 & 563.6 \\
EXP with mean 302.9s & 0.854 & 0.257 & 616.8 \\
\hline GP with mean 202.9s & 0.909 & 0.141 & 515.3 \\
GP with mean 302.9s & 0.854 & 0.230 & 620.0 \\
\hline
\end{tabular}

It can be seen from Table III that the proposed algorithm performs relatively well as it detects traffic anomalies with more than $85 \%$ DR even when mean packet transmission delay ( $302.9 \mathrm{~s}$ ) exceeds time window size. We can see that FAR increases when mean packet transmission delays exceed the time window size because there are not enough microscopic traffic information to distinguish between normal and anomalous traffic patterns. These results again highlights the importance of setting the time window size $L$ to be at least equal to expected mean packet transmission delay.

\section{F. Anomaly classification assessment}

Once an anomaly is detected by a vehicle on a particular freeway segment at time $n_{0}$, a node (a vehicle or a road-side infrastructure) within the freeway segment would continue to gather more information from the vehicles on the segment and monitor the raw decision 
outputs $\left\{o_{n}^{1}, o_{n}^{2}\right\}$ from the MLFFNN until $n_{0}+\eta$ where $\eta$ is the classification interval. For the results presented in this section, $\eta$ is set to $5 \mathrm{~m}$ which is found to give enough classification accuracy in our analysis using the training data set. The classification decision is then made based on the criteria described in Section IV-F.

TABLE IV: Classifications on real-world data from 200 experiments, $\mathrm{CC}=$ Correctly Classified, $\mathrm{MC}=$ Misclassified, $\eta=5$ minutes

\begin{tabular}{|c|c|c|c|}
\hline $\begin{array}{c}\text { Proposed Algorithm } \\
\text { Unlimited } R, \text { PoA }=100 \%\end{array}$ & $\begin{array}{c}\text { Number of } \\
\text { Cases }\end{array}$ & $\begin{array}{c}\text { CC } \\
\text { Cases }\end{array}$ & $\begin{array}{c}\text { MC } \\
\text { Cases }\end{array}$ \\
\hline Transient Anomalies & 9 & 9 & 0 \\
Disruption Precursors & 17 & 17 & 0 \\
\hline Benchmark Algorithm & Number of & CC & MC \\
Cases & Cases & Cases \\
\hline Transient Anomalies & 9 & 6 & 3 \\
Disruption Precursors & 14 & 14 & 0 \\
\hline
\end{tabular}

The classification results in Tables IV and $\mathrm{V}$ are derived based on subset of 200 experiments selected at random where in each experiment, the training and testing sets are selected randomly from the 26 anomaly cases in our real-world data set. In Table IV, anomaly cases are considered as Correctly Classified (CC) if the proposed algorithm can identify their types within the logged duration, i.e. $\left[T_{s}^{a}, T_{s}^{i}\right]$. The Misclassified Cases (MC) shows the number of anomalies that are incorrectly identified by the proposed algorithm. ${ }^{2}$ The proposed algorithm can classify correctly all anomaly cases, while the benchmark algorithm [1] misclassifies three transient anomaly cases as disruption precursors. In fact, these cases were identified as distractions on the shoulder of the freeway due to unexpected appearance of pedestrians in two cases and disabled vehicles in another case (see Fig. 1). These anomaly cases are misclassified by the benchmark algorithm in [1] because the distractions increase the variability of both the relative speed and the covariance between the inter-arrival and inter-departure time which subsequently cause the weighted combination of both temporal and spatial alarms to exceed its classification threshold. In contrast, the algorithm proposed in this paper does not rely on any particular threshold as it only compares the likelihood of the detected anomaly being transient anomaly and the likelihood of being disruption precursor.

We further assess the performance of the proposed algorithm under different $P o A$ coverage and when traffic variables are aggregated according to different percentages of MAC payload availability $R$. It can be seen in

\footnotetext{
2In [1] we refer to "Disruption precursor" as "Incident precursor". For the benchmark algorithm, the three miss-detected cases of incident precursors are excluded from classification evaluation.
}

TABLE V: Classifications on real-world data from 200 experiments with different $P O A$ and percentage of MAC payload availability $(R), \mathrm{CC}=$ Correctly Classified, $\mathrm{MC}=$ Misclassified, $\eta=5$ minutes.

\begin{tabular}{|c|c|c|c|}
\hline Proposed Algorithm & $\begin{array}{c}\text { Number } \\
\text { of Cases }\end{array}$ & $\begin{array}{c}\text { CC } \\
\text { Cases }\end{array}$ & $\begin{array}{c}\text { MC } \\
\text { Cases }\end{array}$ \\
\hline $\begin{array}{c}\text { (Unlimited } R, P o A=50 \%, 100 \%) \\
\text { Transient Anomalies }\end{array}$ & 9 & 9 & 0 \\
Disruption Precursors & 17 & 17 & 0 \\
\hline$(0.5 R, P o A=50 \%, 100 \%)$ & & & \\
Transient Anomalies & 9 & 9 & 0 \\
Disruption Precursors & 17 & 17 & 0 \\
\hline$(0.3 R, P o A=50 \%, 100 \%)$ & & & \\
Transient Anomalies & 9 & 9 & 0 \\
Disruption Precursors & 17 & 15 & 2 \\
\hline
\end{tabular}

Table $\mathrm{V}$ that even when both $P o A$ and MAC payload availability are reduced, all transient anomalies and most of disruption precursors can still be correctly classified. We note that the misclassified cases are disruption precursors that occurred under moderate vehicle density where the persistence of variation of microscopic traffic variables causes the proposed algorithm to misclassify these disruption precursors as transient anomalies.

\section{FINAL REMARKS}

This paper has proposed a novel anomaly classification algorithm that can be deployed in a distributed manner and utilizes microscopic traffic variables shared by neighbouring vehicles to detect and classify traffic anomalies under different traffic conditions. The algorithm uses multi-resolution concepts and is based on the likelihood estimation of the outputs of a feed-forward neural network and a bisection-based decision threshold. The algorithm monitors the change of variability in relative speed, inter-arrival and inter-departure times to capture traffic anomalies. Furthermore, in order to adapt to underlying traffic conditions, the average inter-vehicle spacing is also monitored.

The algorithm's performance on a real-world data test set presents a significant improvement with respect to FAR and DR over the benchmark algorithm proposed in [1]. The preliminary results are very encouraging as they suggest that the algorithm's level of adaptability and resilience to loss of information as a result of low percentage of available vehicles (PoA) or degradation in communications performance is high. For the data set under analysis, it can also detect anomalies on average 13 minutes prior to the occurrence of traffic incidents, therefore significantly increasing the time available to initiate appropriate responses.

There are various aspects worth further consideration. One aspect is to design a model that can incorporate 
information about traffic anomalies from several road links to infer possible impacts on the entire road network. Another aspect worth further investigation is an extension to urban arterial where changes due to traffic signals and its effect in traffic patterns will need to be considered. It would also be interesting, from a traffic management point of view, to classify traffic anomalies according to physical causes (e.g. lane-blocking [9]) to provide more information for incident management. The inclusion of spatial information from other detectors (e.g. cameras on neighbouring segments) in order to locate the bottlenecks and investigate the existence of synchronized flow [36] is also worth investigating. Finally, a more exhaustive assessment of selected VANET technologies and associated protocols in respect to transporting and processing large-scale traffic and sensing information has yet to be assessed in real-world scenarios and is suggested for further investigation.

\section{APPENDIX A}

\section{COMPLEXITY ANALYSIS FOR IMPLEMENTATION}

The main computational complexity of the proposed algorithm consists of the computations (e.g. additions and multiplications) in the DWT and MLFFNN in Figure 3 , while the computational complexity of decision functions for anomaly detection and classification blocks are marginal as they consist mainly of comparisons. The computational complexity of the DWT depends on the input window size $L$ and the number of coefficients in the wavelet and scaling filters $K$. The number of additions and multiplications is originally found to be $2 K(L+K-1)$ using a shift-register in the well-known Pyramid algorithm, but with a more recently proposed method, it is possible to reduce the number of additions and multiplications to $K * L$ [37].

For the MLFFNN block, it is assumed that the proposed algorithm has already been properly trained and the computational complexity is calculated only when the MLFFNN part of the proposed algorithm is downloaded and implemented on a node. The computational complexity of MLFFNN is composed mainly of the number of additions, multiplications and the transfer functions which subsequently depends on the number of neurons in each layer of the neural network. The number of multiplications, additions and the transfer functions as functions of the number of neurons are $m_{0} m_{1}+m_{1} m_{2}+m_{2} m_{3}, m_{1}+m_{2}+m_{3}$ and $m_{1}+m_{2}$ respectively [38] (see Section IV-D for selections of $m_{0}$, $m_{1}, m_{2}$ and $m_{3}$ ).

For the benchmark algorithm [1], the computational complexity depends mainly on the eigenvalue decomposition block while the complexity of the Bayesian change detection and the weighted vote scheme blocks are relatively marginal as they consist mainly of close-form models and comparisons. The eigenvalue decomposition is commonly obtained by implementing Singular Value Decomposition (SVD). Each small eigenvalue in the input window size $L$ can be calculated by performing SVD on a matrix of size $m \times m$, where $m$ is the number of microscopic traffic variable inputs, and it is wellknown that the complexity of computing SVD is $O\left(\mathrm{~m}^{3}\right)$. Therefore, the computation of $L$ small eigenvalues would involve a complexity of approximately $L * O\left(\mathrm{~m}^{3}\right)$.

Our preliminary analysis shows that compared to the benchmark algorithm [1], the computational complexity for implementation of the proposed algorithm is indeed higher due mainly to the computations in MLFFNN. However, a number of experiments in literature has illustrated the feasibility of deploying neural networks in software [21,39].

\section{ACKNOWLEDGMENT}

We would like to thank our anonymous reviewers for their useful comments on previous versions of this paper. This research was partially funded by the National Council of Science and Technology (CONACYT) Mexico. We also would like to thank Dr. Wasan and Dr. Supakorn from NECTEC, Thailand for their assistance in providing the real-world data for our analysis.

\section{REFERENCES}

[1] J. A. Barria and S. Thajchayapong, "Detection and classification of traffic anomalies using microscopic traffic variables," IEEE Transactions on Intelligent Transportation Systems, vol. 12, no. 3, pp. 695-704, September 2011.

[2] V. Alarcon-Aquino and J. A. Barria, "Anomaly detection in communication networks using wavelets," IEE ProceedingCommunications, vol. 148, no. 6, pp. 355-362, December 2001.

[3] V. Chandola, A. Banerjee, and V. Kumar, "Anomaly detection: A survey," ACM Computing Surveys (CSUR), vol. 41, no. 3, pp. 1-58, July 2009, article 15.

[4] C. Oh, J. Oh, and S. G. Ritchie, "Real-time hazardous traffic condition warning system: Framework and evaluation," IEEE Transactions on Intelligent Transportation Systems, vol. 6, no. 3, pp. 265-272, September 2005.

[5] K. Blake, N. Chaudhary, C. Chu, S. Kuchangi, P. Nelson, P. Songchitruksa, D. Swaroop, and V. Tyagi, "Dynamic traffic flow modelling for incident detection and short-term congestion prediction: 1-year progress report," Texas Transport Institute, Texas AM University, Tech. Rep. 0-4946-1, September 2005.

[6] C. Lee, B. Hellinga, and F. Saccomanno, "Real-time crash prediction model for the application to crash prevention in freeway traffic," in CD-ROM, Transportation Research Board of the National Academies, Washington, D.C., 2003, pp. 66-77.

[7] E. Parkany and C. Xie, "A complete review of incident detection algorithms and their deployment: What works and what doesn't," The New England Transportation Consortium, Tech. Rep. 00-7, February 2005. 
[8] Z. Zheng, S. Ahn, D. Chen, and J. Laval, "Freeway traffic oscillations: Microscopic analysis of formations and propagations using wavelet transform,' Transportation Research Part B: Methodological, vol. 45, no. 9, pp. 1378-1388, November 2011.

[9] S. Thajchayapong and J. A. Barria, "Detection of lane-blocking on freeway segments using relative speed and lane changing trajectory," in Proceedings of 9th IEEE International Conference on Intelligent Transportation System Telecommunications, October 2009, pp. 256-261.

[10] T. Nadeem, S. Dashtinezhad, C. Liao, and L. Iftode, "Trafficview: a scalable traffic monitoring system," in Proceedings of IEEE International Conference on Mobile Data Management, 2004, pp. 13-26.

[11] D. Helbing, From Microscopic to Macroscopic Traffic Models. Springer Berlin / Heidelberg, 1998.

[12] B. M. Williams and A. Guin, "Traffic management center use of incident detection algorithms: Findings of a nationwide survey,' IEEE Transactions on Intelligent Transportation Systems, vol. 8 , no. 2, pp. 351-358, June 2007.

[13] L. Iftode, S. Smaldone, M. Gerla, and J. Misener, "Active highways (position paper)," in Proceedings of IEEE 19th International Symposium on Personal, Indoor and Mobile Radio Communications, September 2008, pp. 1-5.

[14] P. Ferrari, "The reliability of the motorway transport system," Transportation Research Part B: Methodological, vol. 22, no. 4, pp. 291-310, August 1988.

[15] J. Sheu, "A sequential detection approach to real-time freeway incident detection and characterization," European Journal of Operational Research, pp. 471-485, 2004.

[16] A. Chen, B. Khorashadi, C. Chuah, D. Ghosal, and M. Zhang, "Smoothing vehicular traffic flow using vehicular-based ad hoc networking and computing grid (vgrid)," in Proceedings of IEEE Intelligent Transportation Systems Conference, 2006, pp. 349 354.

[17] M. Abuelela, S. Olariu, and M. C. Weigle, "Notice: An architecture for the notification of traffic incidents," in Proceedings of IEEE Vehicular Technology Conference, May 2008, pp. 30013005 .

[18] G. K. Mitropoulos, I. S. Karanasiou, A. Hinsberger, F. AguadoAgelet, H. Wieker, H. J. Hilt, S. Mammar, and G. Noecker, "Wireless local danger warning: Cooperative foresighted driving using intervehicle communication," IEEE Transactions on Intelligent Transportation Systems, vol. 11, no. 3, pp. 539-553, September 2010

[19] M. Yongchang, M. Chowdhury, A. Sadek, and M. Jeihani, "Real-time highway traffic condition assessment framework using vehicleinfrastructure integration (vii) with artificial intelligence (ai)," IEEE Transactions on Intelligent Transportation Systems, vol. 10, no. 4, pp. 615-627, December 2009.

[20] L. Wischhof, A. Ebner, and H. Rohling, "Information dissemination in self-organizing intervehicle networks," IEEE Transactions on Intelligent Transportation Systems, vol. 6, no. 1, pp. 90-101, 2005.

[21] M. S. Dougherty and M. R. Cobbett1, "Short-term inter-urban traffic forecasts using neural networks," International Journal of Forecasting, vol. 13, no. 1, pp. 21-31, March 1997.

[22] R. Zito, G. Deste, and M. A. P. Taylor, "Global positioning systems in the time domain: How useful a tool for intelligent vehiclehighway systems?" Transportation Research Part C: Emerging Technologies, vol. 3, no. 4, pp. 193-209, August 1995.

[23] K. Kiratiratanapruk and S. Siddhichai, "Vehicle detection and tracking for traffic monitoring system," in Proceedings of the IEEE TENCON, November 2006, pp. 1-4.

[24] R. L. Cheu and S. G. Ritchie, "Automated detection of laneblocking freeway incidents using artificial neural networks," Transportation Research Part C: Emerging Technologies, vol. 3, no. 6, pp. 371-388, December 1995.

[25] D. Srinivasan, X. Jin, and R. L. Cheu, "Evaluation of adaptive neural network models for freeway incident detection," IEEE Transactions on Intelligent Transportation Systems, vol. 5, no. 1, pp. 1-11, March 2004.

[26] P. C. Pendharkar, "A threshold varying bisection method for cost sensitive learning in neural networks," Expert Systems with Applications, vol. 34, no. 2, pp. 1456-1464, February 2008.

[27] L. Leclercq, "Hybrid approaches to the solutions of the lighthill whitham richards model," Transportation Research Part B: Methodological, vol. 41, no. 7, pp. 701-709, 2007.

[28] H. Adeli and A. Karim, Wavelets in Intelligent Transportation Systems. Wiley, 2005.

[29] K. Hornik, "Multilayer feedforward networks are universal approximators," Neural Networks, vol. 2, no. 5, pp. 359-366, 1989.

[30] H. Dia and G. Rose, "Development and evaluation of neural network freeway incident detection models using field data," Transportation Research Part C: Emerging Technologies, vol. 5, no. 5, pp. 313-331, October 1997.

[31] I. A. Basheer and M. Hajmeer, "Artificial neural networks: fundamentals, computing, design, and application," Journal of Microbiological Methods, vol. 43, no. 1, pp. 3-31, December 2000.

[32] I. Maqsood, M. R. Khan, and A. Abraham, "An ensemble of neural networks for weather forecasting," Neural Computing and Application, vol. 13, no. 2, p. 112122, June 2004.

[33] "Vehicle information and communication system center," http://www.vics.or.jp/english/, Accessed June 2009.

[34] O. Qing, R. Bertini, J. van Lint, , and S. Hoogendoorn, "A theoretical framework for traffic speed estimation by fusing lowresolution probe vehicle data," IEEE Transactions on Intelligent Transportation Systems, vol. 12, no. 3, pp. 747-756, September 2011.

[35] N. An, J. Riihijarvi, and P. Mahonen, "Studying the delay performance of opportunistic communication in vanets with realistic mobility models," in Proceedings of IEEE 69th Vehicular Technology Conference, 2009, pp. 1-5.

[36] B. S. Kerner, The Physics of Traffic: Empirical Freeway Pattern Features, Engineering Applications and Theory. Springer, 2004.

[37] Y. Guo, H. Zhang, X. Wang, and J. R. Cavallaro, "Vlsi implementation of mallat's fast discrete wavelet transformalgorithm with reduced complexity," in IEEE Proceedings of Global Telecommunications Conference, 2001, pp. 320-324.

[38] J. Ahmed, M. N. Jafri, J. Ahmad, and M. I. Khan, "Design and implementation of a neural network for real-time object tracking," Transactions on Engineering, Computing and Technology, vol. 6, pp. 209-212, June.

[39] M. Pasquier and R. J. Oentaryo, "Learning to drive the human way: a step towards intelligent vehicles," International Journal of Vehicle Autonomous Systems, vol. 6, no. 1-2, pp. 24-27, May 2008.

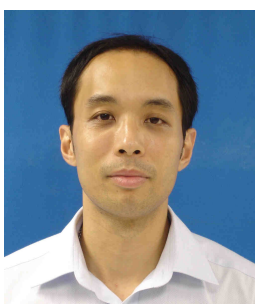

Suttipong Thajchayapong received the PhD degree in electrical and electronic engineering from Imperial College London, London, U.K. He also holds M.S. and B.S. degrees in electrical and computer engineering from Carnegie Mellon University, Pittsburgh, PA, USA. He is currently a Researcher with the National Electronic and Computer Technology Centre (NECTEC), National Science and Technology Development Agency (NSTDA), Thailand. His research interests include intelligent transportation systems with emphasis on vehicular traffic monitoring and inference, anomaly detection, and mobility and qualities of service in wireless networks. 


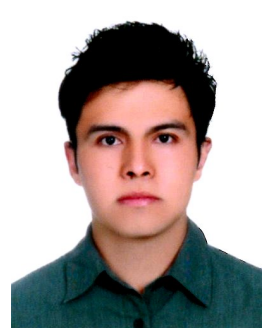

Edgar S. Garcia Trevino received his MS degree in Electronic Engineering (2006) from Universidad de las Americas Puebla, Mexico. He is currently a graduate student at Imperial College London working toward a Ph.D. degree in Electronic Engineering. His current research interest is focused on models for temporal data representation in the context of knowledge discovery and data mining. Mr Garcia was the operation manager of the Atmospheric Monitoring Network of the Puebla State Environment and Natural Resources Agency (in Mexico), from 2001-2008.

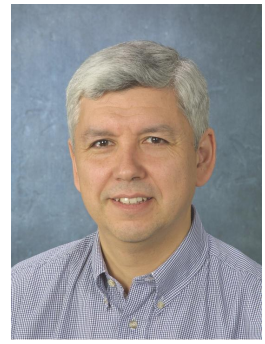

Javier A. Barria (M02) received the Ph.D. degree in electrical and electronic engineering and the MBA degree from Imperial College London, U.K., in 1994 and 1997, respectively. He is a Reader in the Intelligent Systems and Networks Group, Department of Electrical and Electronic Engineering, Imperial College London. His research interests include monitoring strategies for communication and transportation networks, network traffic modelling and forecasting using signal processing techniques, and distributed resource allocation in dynamic topology networks. He has been the joint holder of several European Union Framework and U.K. Engineering and Physical Sciences Research Council project contracts, which are all concerned with aspects of communication systems design and management. Dr. Barria is a Fellow of the Institution of Engineering and Technology and a Chartered Engineer in the U.K. He was a British Telecom Research Fellow from 2001 to 2002 . 